\title{
A note on strong compactness and resurrectibility
}

\author{
by
}

\author{
Arthur W. A pter (New York, NY)
}

\begin{abstract}
We construct a model containing a proper class of strongly compact cardinals in which no strongly compact cardinal $\kappa$ is $\kappa^{+}$supercompact and in which every strongly compact cardinal has its strong compactness resurrectible.
\end{abstract}

1. Introduction and proof of the main theorem. Laver indestructibility [13] in the context of strong compactness has now been the subject of several papers. Readers may consult [2], [1], [4], [5], and [12] for further information on this topic. However, in spite of the research that has been done in this area, there are still many unsolved problems. For instance, in the list of open questions at the end of [1], it is asked whether the first $\alpha$ strongly compact cardinals can be non-supercompact and still exhibit some sort of indestructibility properties, where $\alpha>2$. (The case of $\alpha=2$ is partially discussed in [1], and the case of $\alpha=1$ is discussed in [4].)

The purpose of this note is to provide a partial answer to this question by constructing a model containing a proper class of strongly compact cardinals in which no strongly compact cardinal $\kappa$ is even $\kappa^{+}$supercompact and in which every strongly compact cardinal has its strong compactness resurrectible. Specifically, we prove the following theorem.

THEOREM 1. Let $V \vDash " Z F C+$ There is a proper class of supercompact limits of supercompact cardinals + There are no inaccessible limits of supercompact limits of supercompact cardinals". There is then a partial ordering $\mathbb{P} \subseteq V$ so that $V^{\mathbb{P}} \vDash$ "ZFC + There is a proper class of strongly compact cardinals + No strongly compact cardinal $\kappa$ is $\kappa^{+}$supercompact + Every strongly compact cardinal has its strong compactness resurrectible".

Note that we say a strongly compact cardinal $\kappa$ has its strong compactness resurrectible if after forcing with an arbitrary $\kappa$-directed closed partial

2000 Mathematics Subject Classification: 03E35, 03E55.

Key words and phrases: supercompact cardinal, strongly compact cardinal, indestructibility, resurrectibility. 
ordering $\mathbb{P}$, there is a $\kappa$-distributive partial ordering $\mathbb{Q} \in V^{\mathbb{P}}$ so that $V^{\mathbb{P} * \dot{\mathbb{Q}}} \vDash$ " $\kappa$ is strongly compact". Also, we say that the partial ordering $\mathbb{Q}$ is $\kappa$-distributive if for any ordinal $\alpha<\kappa$, forcing with $\mathbb{Q}$ adds no new $\alpha$ sequences of elements from the ground model.

To prove Theorem 1, let $\left\langle\delta_{\alpha}: \alpha \in\right.$ Ord $\rangle$ enumerate in $V$ the supercompact cardinals together with their measurable limit points. We assume, by doing a preliminary forcing if necessary, that $V \vDash \mathrm{GCH}$.

Let $\mathbb{P}^{0}$ be the partial ordering used in the proof of Theorem 3 of [1]. For completeness, we give the definition of $\mathbb{P}^{0}$ below.

Let $\gamma<\delta$ be so that $\gamma$ is regular and $\delta$ is supercompact. By Lemma 13, pages 2028-2029 of [6] (see also the proof of the Theorem of [2]), there is a $\gamma$-directed closed partial ordering $\mathbb{P}_{\gamma, \delta} \in V$ of rank $\delta+1$ with $\left|\mathbb{P}_{\gamma, \delta}\right|=\delta$ so that $V^{\mathbb{P}_{\gamma, \delta}} \vDash$ "There are no strongly compact cardinals in the interval $(\gamma, \delta)$ since unboundedly many cardinals in $(\gamma, \delta)$ contain non-reflecting stationary sets of ordinals of cofinality $\gamma+$ The cardinal $\delta$ is fully indestructibly supercompact". This has as a consequence that $V^{\mathbb{P}_{\gamma, \delta}} \vDash$ "Any partial ordering not adding bounded subsets to $\delta$ preserves the property that there are no strongly compact cardinals in the interval $(\gamma, \delta)$ ". Further, $\mathbb{P}_{\gamma, \delta}$ is defined as a modification of Laver's indestructibility partial ordering of [13], i.e., as an Easton support iteration of length $\delta$ defined in the style of [13] so that every stage at which a non-trivial forcing is done is a ground model measurable cardinal, the least stage at which a non-trivial forcing is done can be chosen to be an arbitrarily large measurable cardinal in $(\gamma, \delta)$, and at a stage $\alpha$ when a non-trivial forcing $\mathbb{Q}$ is done, $\mathbb{Q}=\mathbb{Q}^{0} * \dot{\mathbb{Q}}^{1}$ where $\mathbb{Q}^{0}$ is $\alpha$-directed closed and $\dot{\mathbb{Q}}^{1}$ is a term for the forcing adding a non-reflecting stationary set of ordinals of cofinality $\gamma$ to some cardinal $\beta>\alpha$. (The exact definition of $\mathbb{Q}^{1}$ can be found in, e.g., [6]. We only note that $\mathbb{Q}^{1}$ is $\gamma$-directed closed.)

We define a proper class Easton support iteration $\mathbb{P}^{0}=\left\langle\left\langle\mathbb{P}_{\alpha}, \dot{\mathbb{Q}}_{\alpha}\right\rangle: \alpha \in\right.$ Ord $>$ as follows:

1. $\mathbb{P}_{1}=\mathbb{P}_{0} * \dot{\mathbb{Q}}_{0}$, where $\mathbb{P}_{0}$ is the partial ordering for adding a Cohen real, and $\dot{\mathbb{Q}}_{0}$ is a term for $\mathbb{P}_{\aleph_{2}, \delta_{0}}$.

2. If $\delta_{\alpha}$ is a measurable limit of supercompact cardinals and $\vdash_{\mathbb{P}_{\alpha}}$ "There is a $\delta_{\alpha}$-directed closed partial ordering so that after forcing with it, $\delta_{\alpha}$ is not $\zeta$ supercompact for some $\zeta$ ", then $\mathbb{P}_{\alpha+1}=\mathbb{P}_{\alpha} * \dot{\mathbb{Q}}_{\alpha}$, where $\dot{\mathbb{Q}}_{\alpha}$ is a term for such a partial ordering of minimal rank which destroys the $\zeta$ supercompactness of $\delta_{\alpha}$ for the minimal possible $\zeta$. By Hamkins' work of [8], [9], and [10], $\zeta$ will be no greater than the degree of supercompactness of $\delta_{\alpha}$ in $V$.

3. If $\delta_{\alpha}$ is a measurable limit of supercompact cardinals and case 2 above does not hold (which will mean that $\vdash_{\mathbb{P}_{\alpha}}$ " $\delta_{\alpha}$ is a measurable limit of supercompact cardinals whose degree of supercompactness is fully indestructible 
and whose strong compactness is fully indestructible"), then $\mathbb{P}_{\alpha+1}=\mathbb{P}_{\alpha} * \dot{\mathbb{Q}}_{\alpha}$, where $\dot{\mathbb{Q}}_{\alpha}$ is a term for the trivial partial ordering $\{\emptyset\}$.

4. If $\delta_{\alpha}$ is not a measurable limit of supercompact cardinals, $\alpha=\beta+1$, $\delta_{\beta}$ is a measurable limit of supercompact cardinals, and case 2 above holds for $\delta_{\beta}$, then inductively, since a direct limit must be taken at stage $\beta,\left|\mathbb{P}_{\beta}\right|=\delta_{\beta}<$ $\delta_{\beta+1}=\delta_{\alpha}$. This means inductively $\mathbb{P}_{\beta}$ has been defined so as to have rank $<\delta_{\alpha}$, so by Lemma 3.1 of [1] and the succeeding remark, $\dot{\mathbb{Q}}_{\beta}$ can be chosen to have rank $<\delta_{\alpha}$. Also, by Lemma 3.1 of [1] and the succeeding remark, $\zeta<\delta_{\alpha}$ for $\zeta$ the least so that $V^{\mathbb{P}_{\beta} * \dot{\mathbb{Q}}_{\beta}}=V^{\mathbb{P}_{\alpha}} \vDash " \delta_{\beta}$ is not $\zeta$ supercompact". Let $\dot{\gamma}_{\alpha}$ be so that $\mathbb{P}_{\mathbb{P}_{\alpha}}$ " $\dot{\gamma}_{\alpha}=\delta_{\beta}^{+}$", and let $\sigma \in\left(\delta_{\beta}, \delta_{\alpha}\right)$ be the least measurable cardinal (in either $V$ or $V^{\mathbb{P}_{\alpha}}$ ) so that $\mathbb{P}_{\mathbb{P}_{\alpha}} " \sigma>\max \left(\dot{\gamma}_{\alpha}, \dot{\zeta}, \operatorname{rank}\left(\dot{\mathbb{Q}}_{\beta}\right)\right)$ ". Then $\mathbb{P}_{\alpha+1}=\mathbb{P}_{\alpha} * \dot{\mathbb{Q}}_{\alpha}$, where $\dot{\mathbb{Q}}_{\alpha}$ is a term for $\mathbb{P}_{\gamma_{\alpha}, \delta_{\alpha}}$ defined so that $\sigma$ is below the least stage at which, in the definition of $\mathbb{P}_{\gamma_{\alpha}, \delta_{\alpha}}$, a non-trivial forcing is done.

5. If $\delta_{\alpha}$ is not a measurable limit of supercompact cardinals and case 4 does not hold, then $\mathbb{P}_{\alpha+1}=\mathbb{P}_{\alpha} * \dot{\mathbb{Q}}_{\alpha}$, where for $\gamma_{\alpha}=\left(\bigcup_{\beta<\alpha} \delta_{\beta}\right)^{+}, \dot{\mathbb{Q}}_{\alpha}$ is a term for $\mathbb{P}_{\gamma_{\alpha}, \delta_{\alpha}}$.

By the proofs of Theorem 3 given in [1] and Hamkins' Theorem 9 given in [5] and the fact that $\mathbb{P}^{0}$ is an iteration of partial orderings satisfying the appropriate degree of directed closure, $V_{1}=V^{\mathbb{P}^{0}} \vDash$ "ZFC + The strongly compact and supercompact cardinals coincide except at measurable limit points + Every supercompact cardinal is fully Laver indestructible + There is a proper class of measurable limits of supercompact cardinals + Every measurable limit $\kappa$ of supercompact cardinals has both its measurability and its strong compactness fully indestructible under $\kappa$-directed closed forcing". Further, by the results in Section 5 of [12], the only measurable limits of supercompact cardinals in $V_{1}$ were supercompact limits of supercompact cardinals in $V$. Therefore, since $V \vDash$ "There are no inaccessible limits of supercompact limits of supercompact cardinals", if $V_{1} \vDash$ " $\kappa$ is the $\alpha$ th measurable limit of supercompact cardinals", $\alpha<\kappa$. As the definition of $\mathbb{P}^{0}$ and the fact that $V \vDash$ GCH ensure that $V_{1} \vDash " 2^{\kappa}=\kappa^{+}$for any measurable limit $\kappa$ of supercompact cardinals (which was a supercompact limit of supercompact cardinals in $V$ )", a result of Menas from [15] immediately yields that $V_{1} \vDash$ "No measurable limit of supercompact cardinals is $2^{\kappa}=\kappa^{+}$supercompact".

Work now in $V_{1}$. Let $\left\langle\sigma_{\alpha}: \alpha \in\right.$ Ord $\rangle$ enumerate the measurable limits of supercompact cardinals. For $\alpha=0$, let $\mathbb{P}_{\alpha}$ be the Easton support iteration of partial orderings which add, for every supercompact cardinal in the interval $\left(0, \sigma_{0}\right)$, a non-reflecting stationary set of ordinals of cofinality $\omega$. For each ordinal $\alpha>0$, let $\mathbb{P}_{\alpha}$ be the Easton support iteration of partial orderings which add, for every supercompact cardinal in the interval $\left(\bigcup_{\beta<\alpha} \sigma_{\beta}, \sigma_{\alpha}\right)$, 
a non-reflecting stationary set of ordinals of cofinality $\left(\bigcup_{\beta<\alpha} \sigma_{\beta}\right)^{+}$. (The precise definition of $\mathbb{P}_{\alpha}$ can be found in [3].) $\mathbb{P}^{1}$ is then defined as the Easton support product $\prod_{\alpha \in \text { Ord }} \mathbb{P}_{\alpha}$.

Take $\mathbb{P}=\mathbb{P}^{0} * \dot{\mathbb{P}}^{1}$. By the standard Easton arguments, $V^{\mathbb{P}} \vDash$ ZFC. By Lemmas $1-6$ of [3] and the succeeding remarks, $V^{\mathbb{P}} \vDash$ "There is a proper class of strongly compact cardinals + No strongly compact cardinal $\kappa$ is $\kappa^{+}$ supercompact". Thus, the proof of Theorem 1 will be complete once we have shown the following.

LEMMA 1.1. $V^{\mathbb{P}} \vDash$ "Every strongly compact cardinal is resurrectible".

Proof. Let $\kappa$ be strongly compact in $V^{\mathbb{P}}=V_{1}^{\mathbb{P}^{1}}$. By Lemmas 1-6 of [3], we know that $\kappa=\sigma_{\alpha}$ for some $\alpha$.

Work again in $V_{1}$. Write $\mathbb{P}^{1}=\mathbb{Q}^{\alpha} \times \mathbb{Q}$, where $\mathbb{Q}^{\alpha}=\prod_{\beta>\alpha} \mathbb{P}_{\beta}$.

Let now $\mathbb{R}$ be a term for a partial ordering in $V^{\mathbb{P}}$ so that $\vdash_{\mathbb{P}}$ " $\mathbb{R}$ is $\kappa$ directed closed", i.e., so that $\Vdash_{\mathbb{P}^{0} *\left(\dot{\mathbb{Q}}^{\alpha} \times \dot{\mathbb{Q}}\right)}$ " $\mathbb{R}$ is $\kappa$-directed closed". Abusing notation somewhat, we can assume without loss of generality that in $V_{1}, \Vdash_{\mathbb{Q}^{\alpha} \times \mathbb{Q}}$ " $\dot{\mathbb{R}}$ is $\kappa$-directed closed". Since $\left(\mathbb{Q}^{\alpha} \times \mathbb{Q}\right) * \dot{\mathbb{R}}$ is a forcing iteration over $V_{1}$, by the Term Forcing Lemma of [11] applied in $V_{1}$ (see also [7] for more information on term forcing), there is a $\kappa$-directed closed partial ordering $\mathbb{R}_{\text {term }} \in V_{1}$ so that forcing with $\mathbb{R}_{\text {term }} \times\left(\mathbb{Q}^{\alpha} \times \mathbb{Q}\right)$ is equivalent to forcing with $\left(\mathbb{Q}^{\alpha} \times \mathbb{Q}\right) * \dot{\mathbb{R}} * \dot{\mathbb{S}}$, where $\dot{\mathbb{S}}$ is a term for a partial ordering.

Rewrite $\mathbb{R}_{\text {term }} \times\left(\mathbb{Q}^{\alpha} \times \mathbb{Q}\right)$ as $\left(\mathbb{R}_{\text {term }} \times \mathbb{Q}^{\alpha}\right) \times \mathbb{Q}$. Since $\mathbb{Q}^{\alpha}$ is $\kappa$-directed closed in $V_{1},\left(\mathbb{R}_{\text {term }} \times \mathbb{Q}^{\alpha}\right)$ is $\kappa$-directed closed in $V_{1}$ as well. Thus, by the relevant indestructibility properties in $V_{1}, V_{1}^{\left(\mathbb{R}_{\text {term }} \times \mathbb{Q}^{\alpha}\right)} \vDash " \kappa$ is a measurable limit of supercompact cardinals". Therefore, the definition of $\mathbb{Q}$ allows Lemma 4 of [3] to be applied here to show $V_{1}^{\left(\mathbb{R}_{\text {term }} \times \mathbb{Q}^{\alpha}\right) \times \mathbb{Q}}=V_{1}^{\mathbb{R}_{\text {term }} \times\left(\mathbb{Q}^{\alpha} \times \mathbb{Q}\right)}=V_{1}^{\left(\mathbb{Q}^{\alpha} \times \mathbb{Q}\right) * \dot{\mathbb{R}} * \dot{\mathbb{S}}}=$ $V_{1}^{\mathbb{P}^{1} * \mathbb{R} * \dot{\mathbb{S}}}=V^{\mathbb{P} * \dot{\mathbb{R}} * \dot{\mathbb{S}}} \vDash " \kappa$ is strongly compact". And, since $\mathbb{R}_{\text {term }} \times \mathbb{Q}^{\alpha}$ is $\kappa$-directed closed, any new $\beta$ sequences for $\beta<\kappa$ present after forcing with the equivalent partial orderings $\left(\mathbb{R}_{\text {term }} \times \mathbb{Q}^{\alpha}\right) \times \mathbb{Q}, \mathbb{R}_{\text {term }} \times\left(\mathbb{Q}^{\alpha} \times \mathbb{Q}\right)$, or $\left(\mathbb{Q}^{\alpha} \times \mathbb{Q}\right) \times$ $\mathbb{R}_{\text {term }}$ must have been added by $\mathbb{Q}$. Hence, as forcing with $\mathbb{R}_{\text {term }} \times\left(\mathbb{Q}^{\alpha} \times \mathbb{Q}\right)$ over $V_{1}$ is equivalent to forcing with $\left(\mathbb{Q}^{\alpha} \times \mathbb{Q}\right) * \dot{\mathbb{R}} * \dot{\mathbb{S}}$ over $V_{1}$, for $\beta<\kappa$, forcing with $\mathbb{S}$ cannot add any new $\beta$ sequences. This completes the proof of Lemma 1.1 .

The proof of Lemma 1.1 completes the proof of Theorem 1.

We conclude this section by making two remarks. First, we observe that by the Lévy-Solovay results [14], the definition of $\mathbb{P}^{1}$ can be redone so that we first add a Cohen real and then define $\mathbb{P}^{1}$ as we did earlier. This changes $\mathbb{P}^{1}$ and $\mathbb{P}^{1} * \dot{\mathbb{R}} * \dot{\mathbb{S}}$, which is equivalent to $\left(\mathbb{Q}^{\alpha} \times \mathbb{Q}\right) \times \mathbb{R}_{\text {term }}$, into a "gap forcing with a gap at $\aleph_{1}$ " in Hamkins' sense of [8], [9], and [10], which, by the results of [8], [9], and [10] again, allows us to infer that after forcing over $V^{\mathbb{P}}=V_{1}^{\mathbb{P}^{1}}$ with $\mathbb{R} * \dot{\mathbb{S}}, \kappa$ has not become $\kappa^{+}$supercompact. Second, if we desire each strongly compact 
cardinal $\lambda$ in our final model to satisfy a weaker form of resurrectibility, i.e., to be so that after forcing with a $\lambda$-directed closed partial ordering $\mathbb{R}$ that does not add subsets to $\lambda$, there is a further partial ordering $\mathbb{S}$ which does not add any new $\lambda$ sequences such that forcing with $\mathbb{S}$ resurrects the strong compactness of $\lambda$, then this is possible to do starting from a model $V \vDash$ "ZFC + There is a proper class of measurable limits of supercompact cardinals + There are no inaccessible limits of measurable limits of supercompact cardinals". We simply use the techniques found in [2] to construct $V_{1}$, beginning by adding a Cohen real, force over $V_{1}$ using the same definition of $\mathbb{P}^{1}$ as used in the proof of Theorem 1, and then apply the methods of Lemma 1.1 to infer the desired form of resurrectibility in our final model. As before, if we start the definition of $\mathbb{P}^{1}$ by adding a Cohen real, since no measurable limit of strongly compact cardinals $\lambda \in V_{1}$ will be $\lambda^{+}$supercompact, we can use the results of [8], [9], and $[10]$ to infer that forcing with $\mathbb{R} * \dot{\mathbb{S}}$ keeps every strongly compact cardinal $\lambda$ as a non- $\lambda^{+}$supercompact cardinal.

2. Concluding remarks. In conclusion to this note, we ask if any of the strongly compact cardinals in our final model $V^{\mathbb{P}}$ is fully indestructible, or indeed, if there is any way of constructing a model containing a proper class of strongly compact cardinals in which no strongly compact cardinal $\kappa$ is $\kappa^{+}$ supercompact yet all strongly compact cardinals $\kappa$ have their strong compactness indestructible in some variation of the usual sense of indestructibility. We conjecture that the answer to the latter of these two questions is yes.

\section{References}

[1] A. Apter, Aspects of strong compactness, measurability, and indestructibility, Arch. Math. Logic, submitted.

[2] - Laver indestructibility and the class of compact cardinals, J. Symbolic Logic 63 (1998), 149-157.

[3] —, Patterns of compact cardinals, Ann. Pure Appl. Logic 89 (1997), 101-115.

[4] A. Apter and M. Gitik, The least measurable can be strongly compact and indestructible, J. Symbolic Logic 63 (1998), 1404-1412.

[5] A. Apter and J. D. Hamkins, Universal indestructibility, Kobe J. Math. 16 (1999), 119-130.

[6] A. Apter and S. Shelah, Menas' result is best possible, Trans. Amer. Math. Soc. 349 (1997), 2007-2034.

[7] J. Cummings, A model in which GCH holds at successors but fails at limits, ibid. 329 (1992), 1-39.

[8] J. D. Hamkins, Destruction or preservation as you like it, Ann. Pure Appl. Logic 91 (1998), 191-229. 
[9] J. D. Hamkins, Gap forcing, Israel J. Math., to appear.

[10] - Gap forcing: generalizing the Lévy-Solovay theorem, Bull. Symbolic Logic 5 (1999), 264-272.

[11] - Small forcing makes any cardinal superdestructible, J. Symbolic Logic 63 (1998), 51-58.

[12] -, The lottery preparation, Ann. Pure Appl. Logic 101 (2000), 103-146.

[13] R. Laver, Making the supercompactness of $\kappa$ indestructible under $\kappa$-directed closed forcing, Israel J. Math. 29 (1978), 385-388.

[14] A. Lévy and R. Solovay, Measurable cardinals and the continuum hypothesis, ibid. 5 (1967), 234-248.

[15] T. Menas, On strong compactness and supercompactness, Ann. Math. Logic 7 (1974), 327-359.

Department of Mathematics

Baruch College of CUNY

New York, NY 10010, U.S.A.

Web: http://math.baruch.cuny.edu/ apter

E-mail: awabb@cunyvm.cuny.edu

Received 1 February 2000 